SLAC-PUB-3243

LBL- 16824

October 1983

(N)

\title{
STUDY OF HEAVY QUARK PRODUCTION WITH THE MARK II AT PEP*
}

G. Abrams, D. Amidei, A. Baden, C. de la Vaissiere, G. Gidal, M. Gold, G. Goldhaber, L. Golding, D. Herrup, I. Juricic, J. Kadyk, M. Nelson, P. Rowson, H. Schellman, P. Sheldon, and G. Trilling

\section{Lawrence Berkeley Laboratory \\ University of California \\ Berkeley, California 94720}

A. Boyarski, M. Breidenbach, P. Burchat, D. Burke, J. Dorfan, L. Dunn, G. Feldman, L. Gladney, G. Hanson, R. Hollebeek, W. Innes, J. Jaros, M. Jonker, S. Klein, A. Lankford, R. Larsen, B. LeClaire, N. Lockyer, V. Lüth, C. Matteuzzi, R. Ong, M. Perl, N. Phinney, J. Price, B. Richter, M. Ross, D. Schlatter, J. Smith, J. Yelton, and C. Zaiser

Stanford Linear Accelerator Center Stanford, California 94305

C. Blocker, M. Eaton, M. Levi, R. Schwitters, and T. Schaad

Harvard University

Cambridge, Massachusetts 02138

Presented at the 7 th International Conference on Meson Spectroscopy, Upton, New York, April 14-16, 1983.

\footnotetext{
* This work was supported in part by the Director, Office of Energy Research, Division of High Energy and Nuclear Physics of the U.S. Department of Energy under Contracts DE-AC03-76SF00098 and DE-AC03-76SF00515.
} 


\title{
STUDY OF HEAVY QUARK PRODUCTION WITH THE MARK II AT PEP
}

\author{
G.S. Abrams \\ Lawrence Berkeley Laboratory, University of California, Berkeley, CA 94720
}

\begin{abstract}
The methods adopted by the Mark II collaboration to study heavy quark production at PEP are described. Two complementary techniques are used: $D^{*}$ tagging using the decay chain $D^{\circ+} \rightarrow D^{0} \pi^{+}, D^{0} \rightarrow K^{-} \pi^{+}$, and inclusive lepton tagging using the characteristic $p_{T}$ distributions to distinguish contributions from $b$ and $c$ quarks. These techniques are used to derive information about heavy quark fragmentation and about the weak coupling of heavy quarks.
\end{abstract}

\section{INTRODUCTION}

A study of heavy quark production in $\mathrm{e}^{+} \mathrm{e}^{-}$annihilation yields information on the couplings of the quarks, on their hadronization, and on their weak decays. In this talk methods will be discussed which allow the identification of data samples enriched in $b$ and $c$ quark production. These samples are then used to study $b$ and $c$ fragmentation, and to search for a possible forward-backward asymmetry in the $b / c$ production angular distributions.

\section{THE MARK II DETECTOR}

Shown in Fig. 1 is a schematic view of the Mark II detector at PEP. The detector elements important for the analysis to be presented below are the cylindrical drift chamber, the eight lead-liquid argon (LA) electromagnetic calorimeters, and the proportional tubes interspersed with the iron flux return and with iron hadron absorber to furnish muon identification.

Charged particle momenta are measured in a 4.6 kilogauss solenoidal magnetic field by a 16 layer drift chamber ${ }^{1}$ subtending $80 \%$ of the solid angle. The momentum resolution is

$$
\left(\frac{\delta p}{p}\right)^{2}=(0.015)^{2}+(0.01 p)^{2}
$$

where the momentum $\mathrm{p}$ is in $\mathrm{GeV} / \mathrm{c}$. The LA calorimeters ${ }^{2}$ are used for $\pi / \mathrm{e}$ discrimination, and will be described in more detail below. The muon detection system consists of four layers of irôn absorber interleaved with proportional tube counters. Above $2 \mathrm{GeV} / \mathrm{c}$ pions are misidentified as muons (either through decay or punch through) with a probability of $\approx 1 \%$.

\section{EVENT SELECTION}

The hadronic event sample was selected using the criteria:

1) a primary vertex reconstructed near the interaction point $(4 \mathrm{~cm}$ radially and $7 \mathrm{~cm}$ in z),

2) 5 or more well-measured charged tracks,

3) visible energy (charged momentum plus $L A$ energy for neutrals) $>E_{c m} / 4$, and

\footnotetext{
The members of the Mark 11 collaboration are: G. Abrams, D. Amidei, A Baden, C. de la Vaissierre, G. Gidal, M. Gold, G. Goldhaber, L. Goldine, D. Herrup, I. Juricic, J. Kadyk, M. Nelson, P. Rowson, H. Schellman, P. Sheldon, and G. Trilling, Lawrence Berkeley Laboratory, University of California, Berkeley, CA 94720, A Boyarsk, M. Breidenbach, P. Burchat, D. Burke, J. Dorfan, L Dunn, G. Fetdman, L Gladney, G. Hanson, R. Hollebeek, W. Innes, J. Jaros, M. Jonker, S. Klein, A Lankford, R. Larsen, B. LeClaire, N. Lockyer, V. Lith, C. Matteuzzi, R. Ong, M. Perl, N. Phinney, J. Price, B. Richter, M. Ross, D. Schlatter, J. Smith, J. Yelton, and C. Zaiser, Stanford Linear Acoelerator Center, P.O. Box 4349, Stanford, CA 94305; and C. Blocker, M. Eaton, M. Levi, R. Schwitters, and T. Schaad, Harvard University, Cambridge, MA 02138.
} 


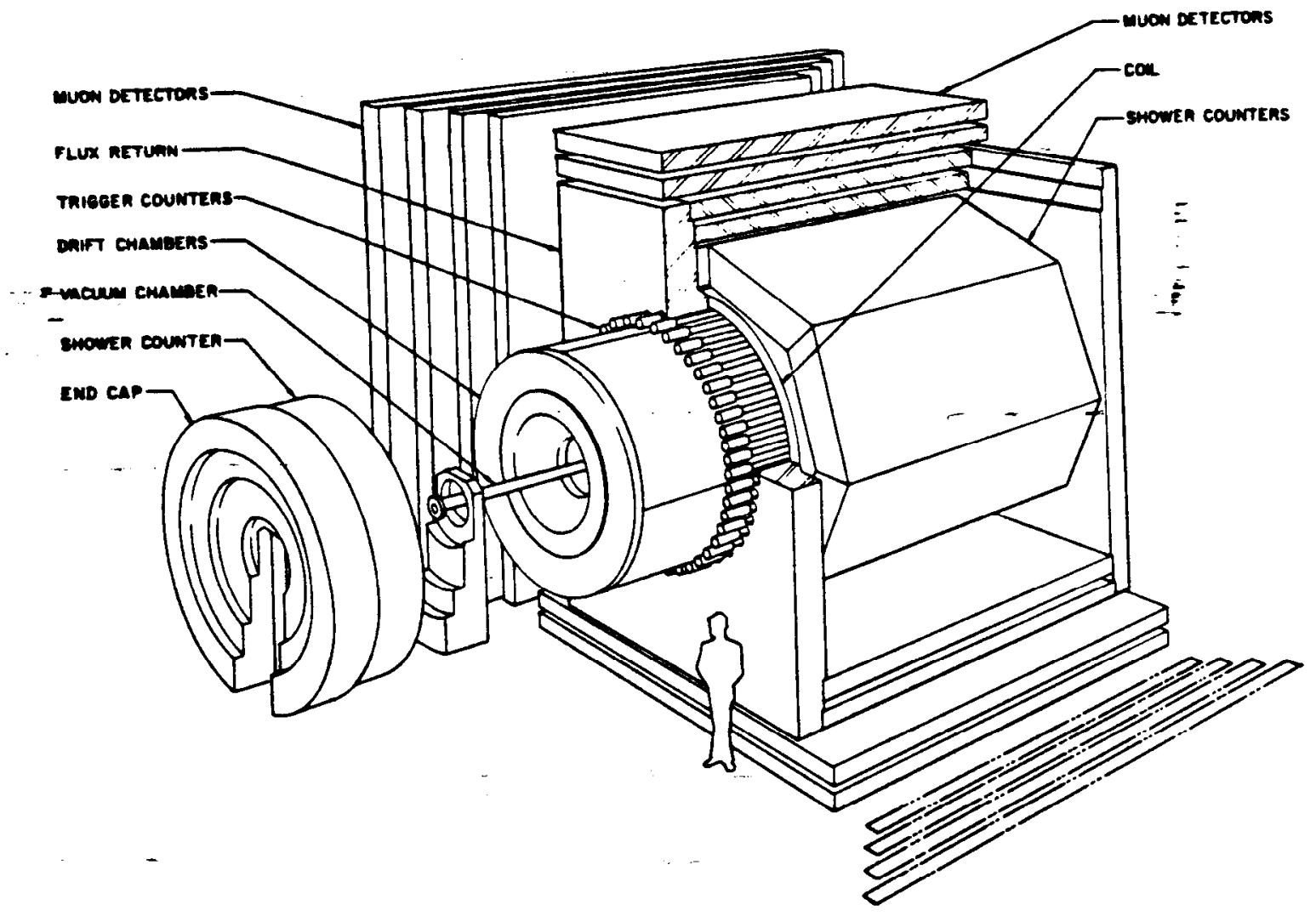

Fig. 1. Schematic view of the Mark II detector at PEP.

4) $\left|\cos \theta_{\text {thrust }}\right|<0.7$

A well-measured charged track was subject to the requirements:

1) radial distance of closest approach to the interaction point $<4 \mathrm{~cm}$, and $8 \mathrm{~cm}$ in 2 ,

2) $p \sin \theta>0.1 \mathrm{GeV}$ and $p<16 \mathrm{GeV}$, and

3) :- not be a possible product of a gamma pair conversion or $\pi^{0}$ Dalitz decay.

The neutral energy received contributions from reconstructed photons in the LA which:

1) have $>0.2 \mathrm{GeV}$ total energy,

2) share no more than $50 \%$ of their energy with other tracks,

3) lie within the fiducial volume of the LA ( 2 strips inside of the physical edges), and

4) are more than $7 \mathrm{~cm}$ distant from the closest charged track.

\section{$D^{\bullet}$ RECONSTRUCTION}

The search for a charm signal at PEP using effective masses of combinations such as $\mathrm{K}^{-} \pi^{+}, \mathrm{K}_{1}^{0} \pi^{+}, \mathrm{K}^{-} \pi^{+} \pi^{+}$, and $\mathrm{K}^{-} \pi^{+} \pi^{+} \pi^{-}$has thus far not been successful due to a large combinatoric background and inadequate resolution. A technique to identify charmed events developed at SPEAR $R^{3}$ using the decay $D^{+} \rightarrow D^{0} \pi^{+}$circumvents these difficulties. (In this report when specific charge states are referenced, the use of the charge conjugate state is implied as well.) Figure 2 shows the distribution of $\Delta$, where 


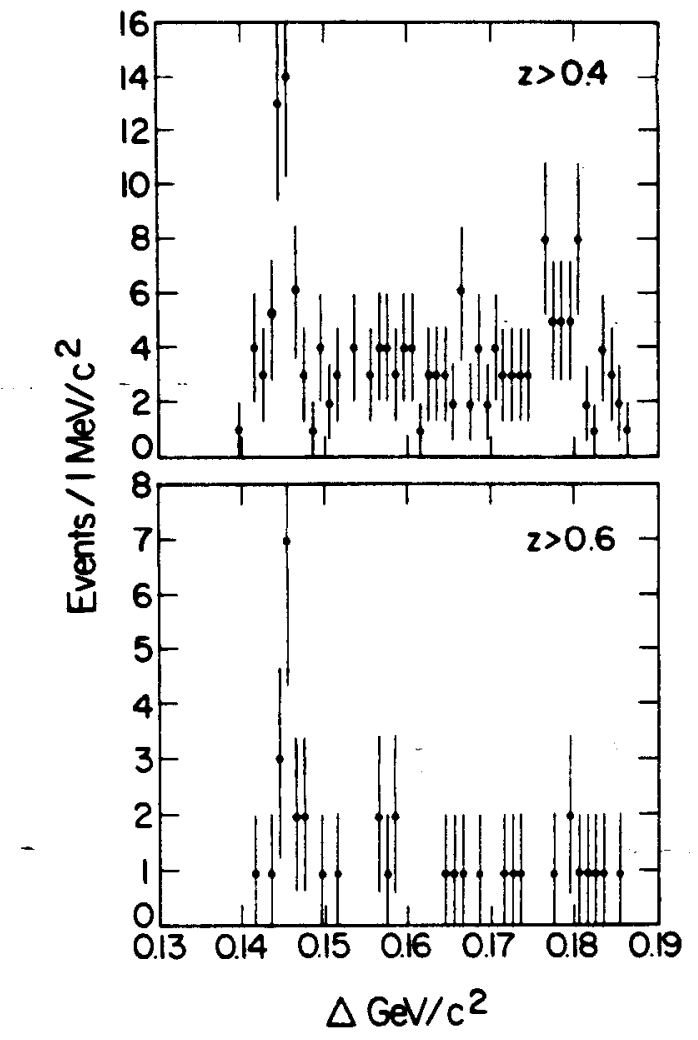

Fig. 2. Distribution of $\Delta$ for $D^{0}$ events at SPEAR.

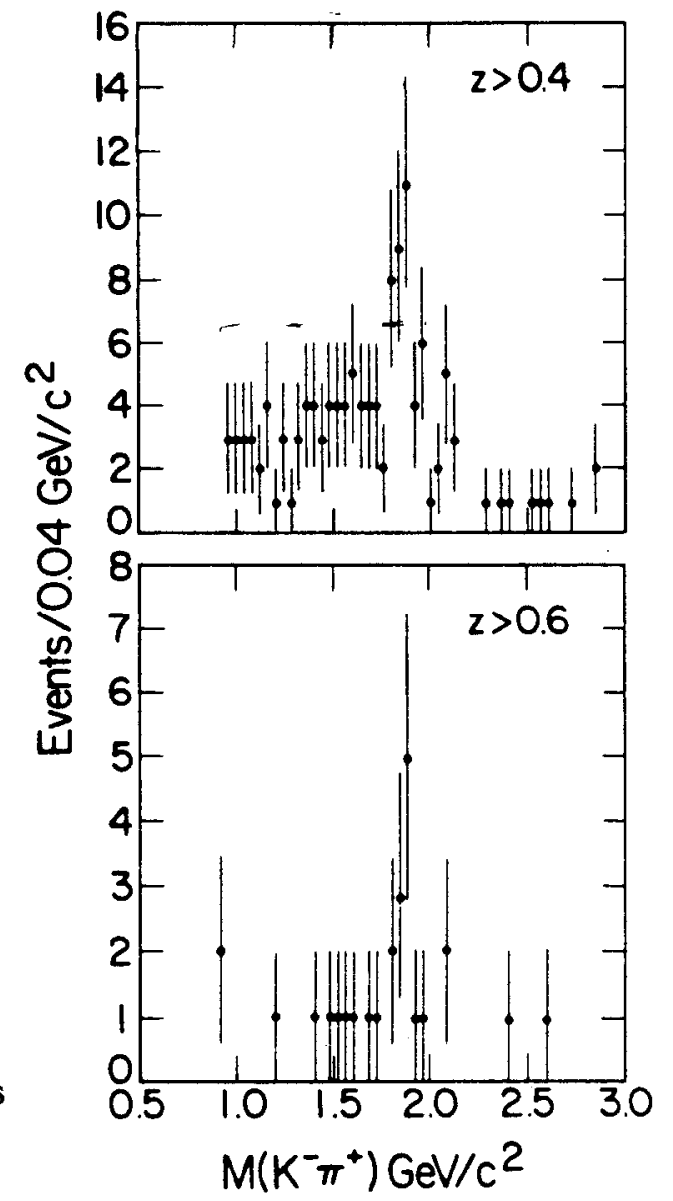

Fig. 3. Distribution of $M\left(K^{-} \pi^{+}\right)$for $D^{*}$ candidates at SPEAR.

$$
\Delta=M\left(D^{0} \pi^{+}\right)-M\left(D^{0}\right)
$$

for $\mathrm{D}^{0}$ candidates with

$$
1.76<\mathrm{M}\left(\mathrm{K}^{-} \pi^{+}\right)<1.96 \mathrm{GeV} / \mathrm{c}^{2}
$$

and where $z_{D^{*}}=2 E_{D^{*}} / \sqrt{s}$. A clear signal near threshold due to the $D^{*}$ is apparent. This argument may be inverted, using a cut on $\Delta$,

$$
143<\Delta<147 \mathrm{MeV} / \mathrm{c}^{2}
$$

and then plotting the $\mathrm{K}^{-} \pi^{+}$effective mass; Fig. 3 shows a clear $D^{0}$ signal. These $D^{*}$ events will be used below to study $c \rightarrow D^{\prime \prime}$ fragmentation.

As Figs. 2 and 3 illustrate, the price one pays for the cleanliness of the $D^{*}$ signal is in statistics. In an effort to increase the yield of $D^{*}$ events, necessary for the measurement of the electroweak interference of the $c$, we return to a study ${ }^{4}$ of SPEAR data. Figure 4 shows the $\mathrm{K}^{+} \pi^{+}$effective mass distribution at several center of mass energies. Besides the expected $D^{0}$ signal at $1.86 \mathrm{GeV}$ there is a satellite enhancement, broader and at lower mass $(\sim 1.6 \mathrm{GeV})$. This enhancement, here called the $S^{0}$, has a mass and width that are independent of the beam energy, as illustrated in Fig. S. The $S^{0}$ does not appear in the data at the $\psi$ or $\psi$ (not shown), and is therefore associated with the charm threshold, arising from the $D^{0}$ or $D^{+}$. 


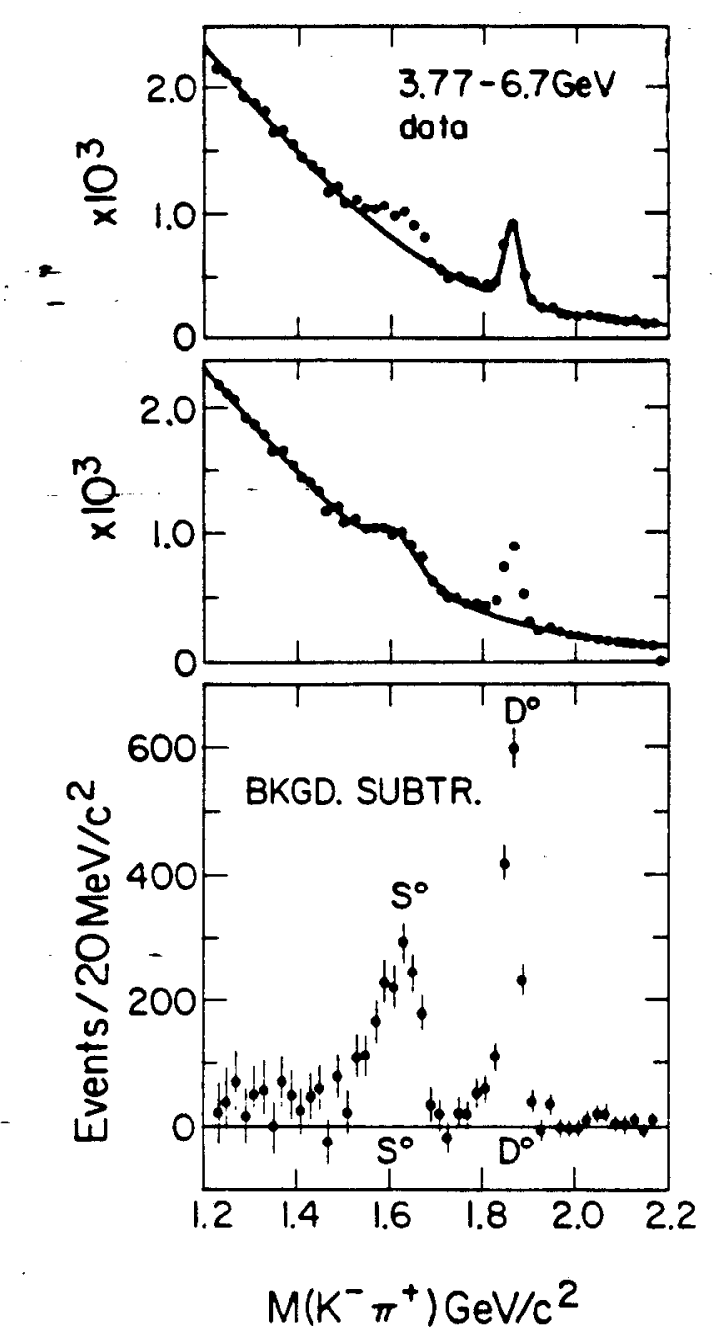

Fig. 4. $\mathrm{K}^{-} \pi^{+}$mass distribution at SPEAR.

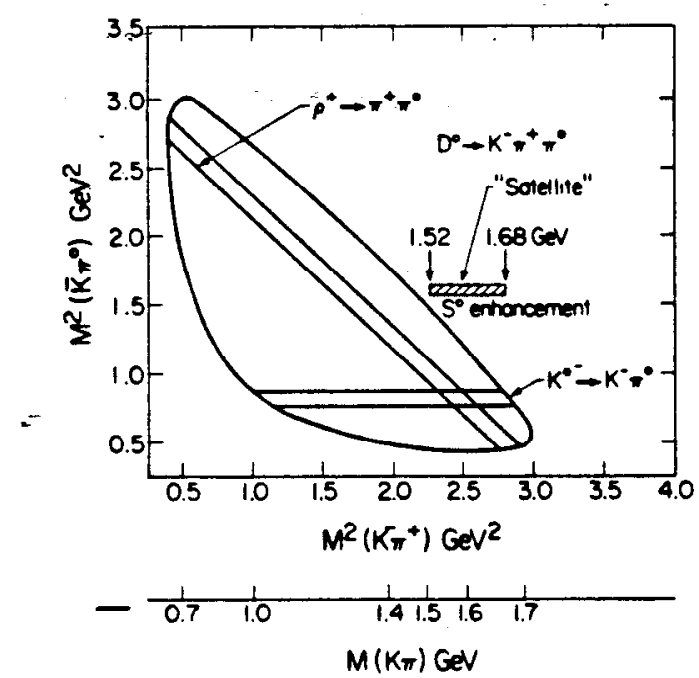

Fig. 6. Schematic representation of the $K^{-} \pi^{+} \pi^{0}$ Dalitz plot at the $D^{0}$.

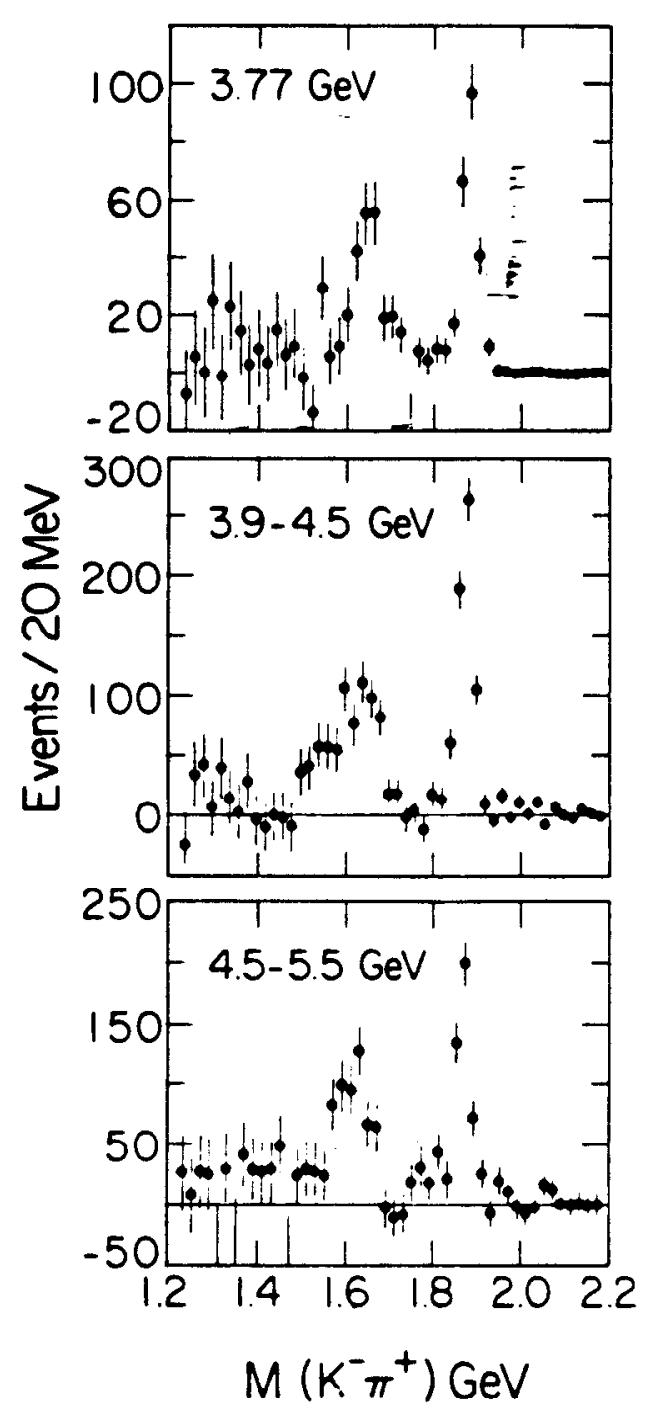

Fig. 5. $\boldsymbol{K}^{-} \pi^{+}$mass distribution at several SPEAR energies.

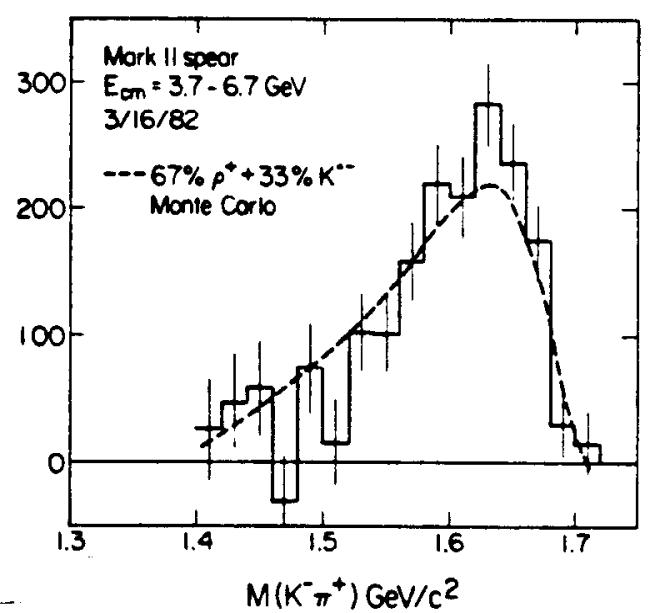

Fig. 7. Monte Carlo simulation of an $S^{0}$ peak. 
An understanding of the $S^{0}$ is aided by an inspection of the $\mathrm{D}^{0} \rightarrow \mathrm{K}^{-} \mathrm{\rho}^{+} \rightarrow \mathrm{K}^{-} \pi^{+} \pi^{0}$ Dalitz plot (and with this analysis I believe I have earned my admission to a conference on meson spectroscopy), Fig. 6. The $\rho^{+}$is aligned purely in the $m=0$ state since the $D^{0}$ is $\mathrm{J}^{p}=0^{-}$, and therefore produces a $\cos ^{2} \theta$ distribution between the $\mathrm{K}^{-}$direction and the $\pi^{+}$direction in the $\rho^{+}$rest frame. The forward $\pi^{+1}$ s give rise to the $S^{0}$ (with very low energy $\pi^{0} s$ ); backward $\pi^{+1} s$ are expected to give rise to a low mass $\mathrm{K}^{-} \pi^{+}$enhancement. Figure 7 shows that this interpretation (and a little $\mathrm{K}^{*} \pi^{+}$) explains the shape of the $S^{0}$ peak. This $S^{0}$ enhancement, which is comparable to the $\mathrm{D}^{0} \rightarrow \mathrm{K}^{-} \pi^{+}$yield, is thus an additional convenient signature for charmed mesons.

\section{ELECTRON IDENTIFICATION}

In hadronic events a large charged particle multiplicity ( $\sim 12$ per event) and a small ( $\sim 10$ percent) semi-leptonic decay rate for heavy quarks yields an average signal to background ratio of $\sim 1 / 300$ for electrons from b decay with $p>1 \mathrm{GeV} / \mathrm{c}$ to all charged hadrons. (These hadrons are dominantly pions; for our discussion the distinction will not be crucial and we will use the terms interchangeably.)

The basis for electron identification with the Mark II is a close interplay of drift chamber and LA information. The charged tracking data is used both to establish an expected event-by-event energy normalization based on the measured momentum $p$, and to determine a narrow road through the LA for the collection of LA pulse height to be associated with the charged track. The geometry of the LA strips is illustrated in Fig. 8; energy is collected separately from the three coordinates $F, T$ and $U$. As an example, Fig. 9 shows the U-strip energy, $E_{U}$, for electrons (Fig. 9(a)) and pions (Fig. 9(b)) normalized by the measured momentum for tracks detected at the $\psi$. The cut at $E_{\mathrm{U}} / \mathrm{p}$ indicated on Fig. 9 at 0.1 is chosen to yield a high efficiency for electron acceptance and simultaneously a low probability for misidentification of pions (the cuts for the various coordinates are chosen to equalize the discrimination power of each measurement).

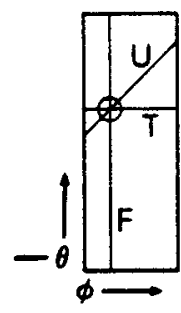

Fig. 8.

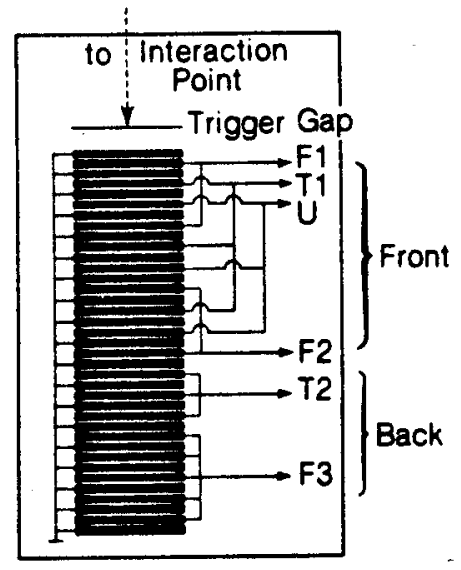

Schematic representation of the geometry of the LA strips and the ganging in depth.

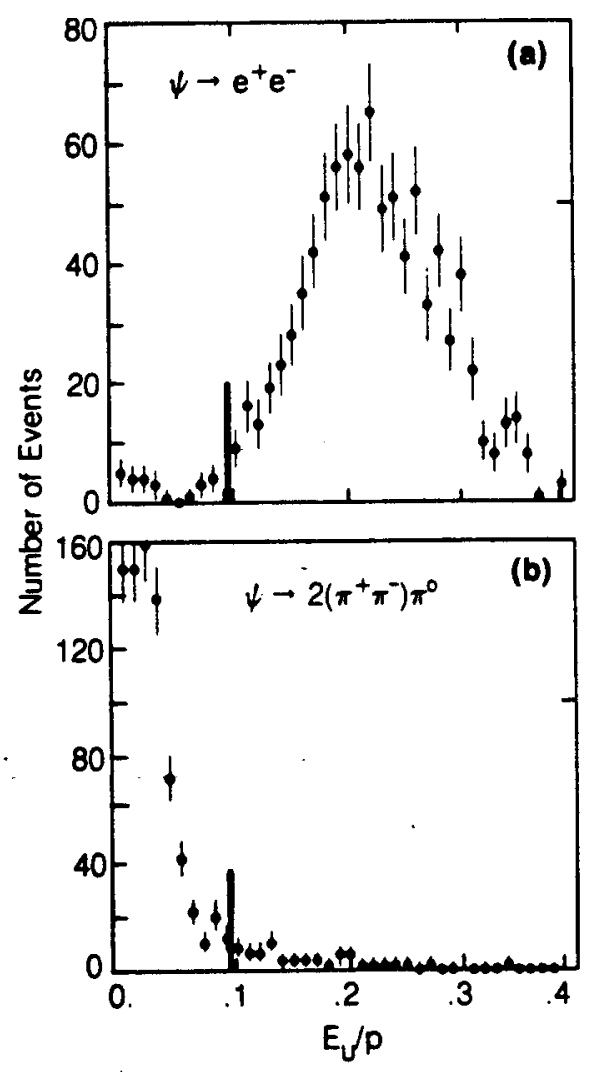

Fig. 9. Distribution of the normalized $u$-strip energy $E u / p$ for a) electrons, b) pions.

Finally, a test quantity, TEST1, is defined as the minimum of the quantities

$$
\begin{gathered}
E_{\mathrm{F}} / 0.14 \mathrm{p} \\
\mathrm{E}_{\mathrm{T}} / 0.10 \mathrm{p} \\
\mathrm{E}_{\mathrm{U}} / 0.10 \mathrm{p} \\
\text { or }\left(\mathrm{E}_{\text {front }}=\mathrm{E}_{\mathrm{F}}+\mathrm{E}_{\mathrm{T}}+\mathrm{E}_{\mathrm{U}}\right) / 0.5 \mathrm{p}
\end{gathered}
$$




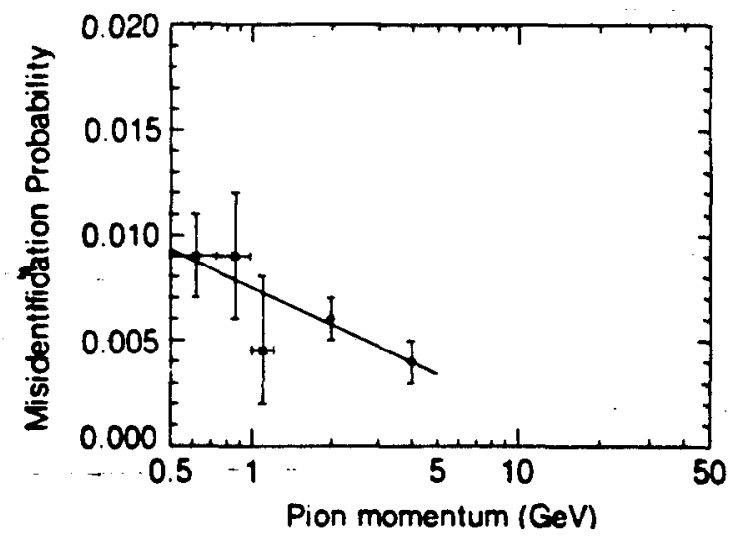

Fig. 10. Measured pion misidentification as a function of momentum.

An electron is then defined as a track with TEST1 $>1.1$, and a pion one with TEST1 < 1.1. This strategy may be seen to discriminate against non-interacting pions or interacting pions with a small energy deposition near the track in any measured coordinate $F, T$, or $U$; the test on the "Front"

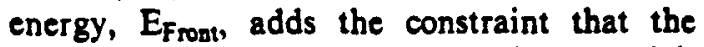
energy of a typical electron usually materializes in the first eight radiation lengths of the LA system. Figures 10 and 11 show the measured pion misidentification probability and electron efficiency, respectively, as a function of $p$. The identification algorithm is summarized in Fig. 12.

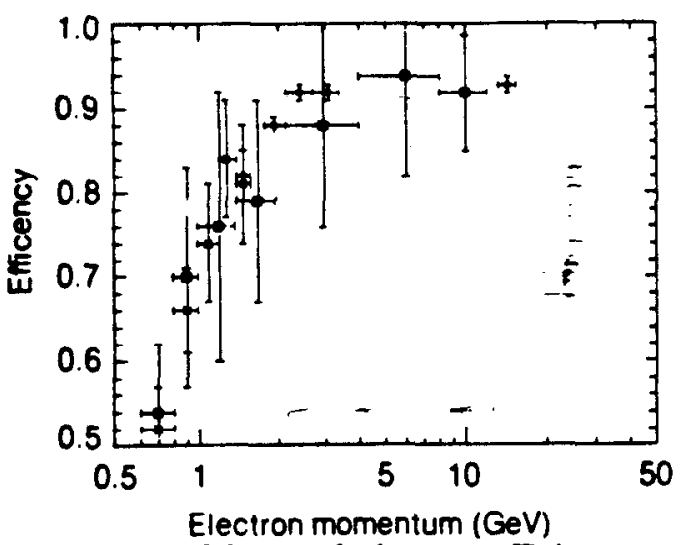

Fig. 11. Measured electron efficiency as a function of momentum.

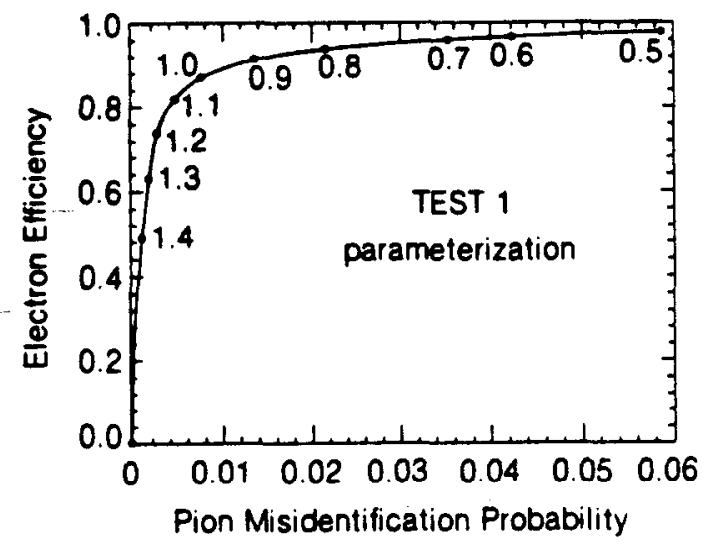

Fig. 12. Electron efficiency as a function of pion misidentification proba. bility for various values of TEST1.

\section{BACKGROUND PROCESSES}

In addition to the misidentification problem discussed above, several other background processes contribute real electron signals which do not originate from heavy quark semi-leptonic decays. A major source of background is from photon conversions in the material surrounding the beam pipe. To search for such conversions, all electron candidates are combined with oppositely charged tracks to ascertain if the electron is compatible with arising from a pair conversion. Geometrical cuts are then used to select pairs of tracks which are close in space with a small opening angle. For these pairs where the second number has a LA response not in contradiction with the electron hypothesis, the reconstructed radius of conversion is shown in Figs. 13(a) and 13(b) (for two different configurations of the PEP beam pipe and close-in detectors). Evident in the figure are the images of the material surrounding the beam.

Electrons from identified conversions are eliminated from the direct lepton sample. Since the pair-finding efficiency is only $\sim 80 \%$ (due to reconstruction difficulty when the high momentum $\mathrm{e}^{+} e^{-}$share drift chamber hits), there is a residual contamination due to this source. Using the Monte Carlo to measure this efficiency, we correct the direct lepton sample for this backeround.

Other sources of contamination arise from higher order QED processes, two of which are illustrated in Fig. 14. Though in the usual Mark $H$ analysis events from these processes are rejected as hadronic candidates via a cut on the observed energy, in our case the $e^{ \pm}$are observed at large $q^{2}$ and contribute to the total energy sum. A hand-scan of all events with electron momentum above $6 \mathrm{GeV} / \mathrm{c}$ was made to study the background. Figure 15 shows an event 


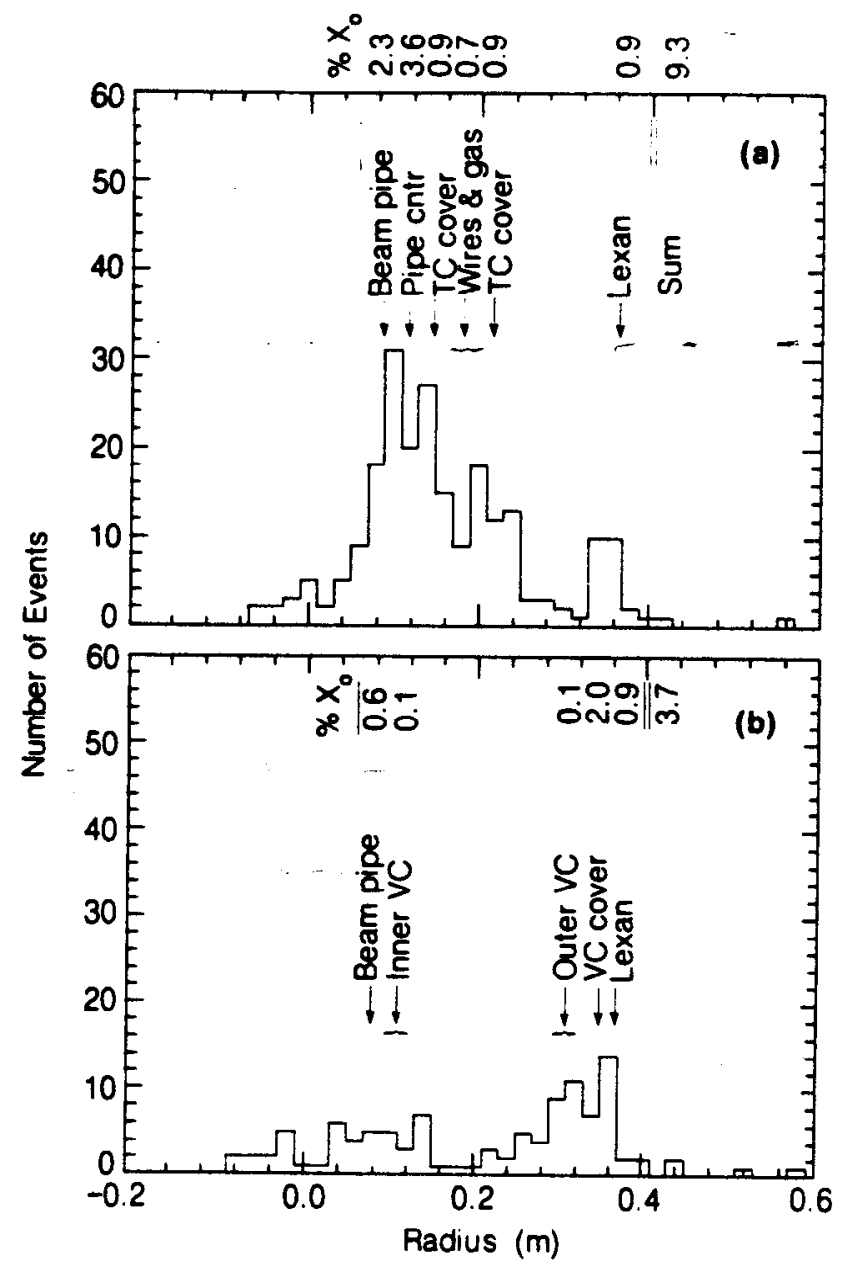

Fig. 13. Reconstructed radius of conversion for electron pair candidates. a) Old Trigger Chamber (TC) data. b) New Vertex Chamber (VC) data.

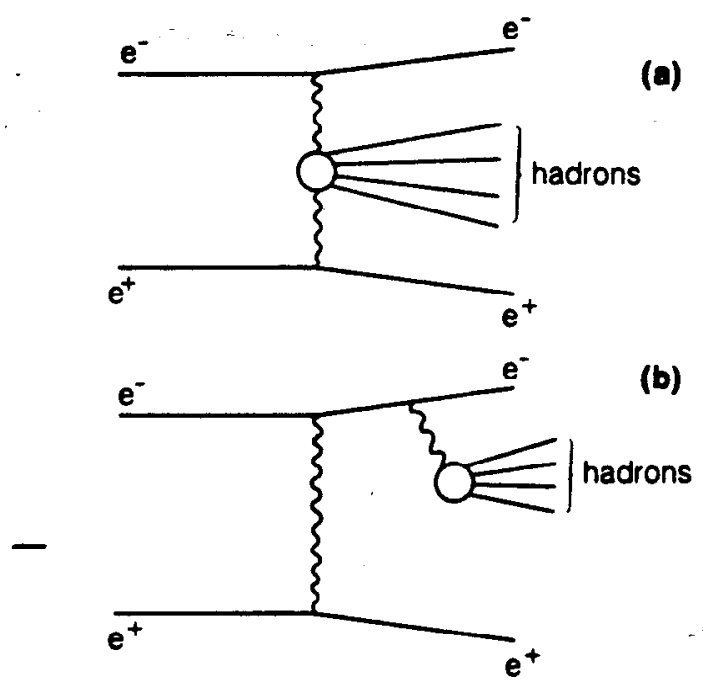

Fig. 14. Higher order QED processes leading to observable high energy electrons.

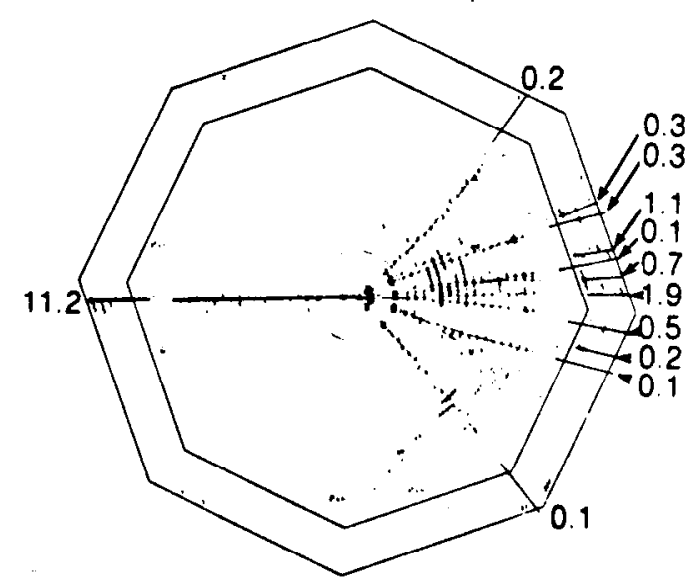

Fig. 15. Multi-hadronic event with a high energy isolated track (darkened track). The associated LA pulse heights (in GeV) are shown around the border of the LA octants. 
from the scan identified as a "typical" QED background event. Events with identified isolated electrons as in Fig. 15 are eliminated in the hand scan. A Monte Carlo calculation predicts 8-16 such higher-order QED events, compared with the 17 events actually found and removed.

\section{FIT TO INCLUSIVE $e^{ \pm}$DISTRIBUTIONS}

A total of 10,691 events passed the event selection criteria above. In Table Ifs shown the raw "prompt" signal of all identified electron candidates in accepted hadronic events (identified gamma conversions and Dalitz pairs have been eliminated here) for bins in the electron momentum, p, and momentum transfer, $p$, with respect to the event thrust axis. The charged tracks in these events which satisfy all the electron identification criteria except the pulse height test (i.e., TESTl < 1.1) are the next entry in this table. From the measured pion misidentification probability (determined as a function of $p$ and $p_{T}$ ), the bin-by-bin number of misidentified pions is calculated. The contribution to the raw signal from non-identified pair conversions and Dalitz decays is estimated from the Monte Carlo efficiencies.

The various backgrounds are subtracted from the raw signals on a bin-by-bin basis. Then each bin is corrected for detection efficiency, including effects from both event selection criteria and electron identification. Error estimates, both statistical and systematic, conclude the entries in Table I. The systematic errors are dominated by effects due to pion misidentification (estimated as $-50 \%$ independent of p, p), and those due to other backgrounds, mainly pair contamination (estimated as $-30 \%$ ).

The measured rate for inclusive electron production for $p>1 \mathrm{GeV} / \mathrm{c}$ is

$$
0.056 \pm 0.006 \pm 0.040
$$

electrons per hadronic event. This is a relatively model independent result which is only slightly sensitive to the uncertainty in the electron acceptance.

Further analysis of the data depends more on the Monte Carlo simulation, particularly on the production model and on the models for semi-electronic decay for $b$ and $c$ quarks. The heavy quark fragmentation is parameterized in a form ${ }^{5}$ now familiar to this Conference:

$$
D_{Q}^{H}(z) \sim \frac{1}{z\left[1-\frac{1}{z}-\frac{\epsilon_{Q}}{1-z}\right]^{2}} .
$$

Here the fragmentation of the heavy quark $Q$ into the heavy meson $H$ has a shape which is a function only of the parameter $\epsilon_{Q}$ (see Fig. 16). A kinematical argument ${ }^{5}$ leads us to expect

$$
\epsilon_{Q} \sim 1 / \mathrm{m} \xi
$$

The semi-leptonic decays of B and D mesons are modeled to agree with the CLEO 6 and DELCO 7 data.

The bins in the p-pT plane can then be fit with the parameters

$$
\begin{aligned}
& \mathrm{BR}_{\mathrm{c}} \mathrm{c} \rightarrow \mathrm{e} \text { branching ratio } \\
& \mathrm{BR}_{\mathrm{b}} \mathrm{b} \rightarrow \mathrm{c} \text { branching ratio } \\
& \epsilon_{c} \quad \text { fragmentation shape parameter for } \mathrm{c} \text { production } \\
& \epsilon_{b} \quad \text { fragmentation shape parameter for } b \text { production. }
\end{aligned}
$$

With $\epsilon_{c}$ fixed at 0.3 (see next section), a good fit with a $\chi^{2}$ of 14.7 for 18 degrees of freedom is achieved with the values

$$
\begin{aligned}
& \mathrm{BR}_{c}=0.066 \\
& \mathrm{BR}_{b}=0.105 \\
& \mathrm{C}_{b}=0.028
\end{aligned}
$$

We thus see evidence for a peaking of the $b$ fragmentation function at large $z$. The average value of $z$ from this analysis (and also from another parameterization of the form $z^{\alpha}(1-z)$ ) is 
Table I. Electron signal and background

\begin{tabular}{|c|c|c|c|c|c|}
\hline$p \backslash p_{T}$ & 0.0 .5 & $0.5-1.0$ & $1.0-1.5$ & $>1.5$ & \\
\hline $1-2$ & $\begin{array}{c}363 \\
10952 \\
241 \pm 88 \\
66 \\
102 \\
47 \\
196 \\
\end{array}$ & $\begin{array}{c}112 \\
5662 \\
57 \pm 28 \\
12 \\
79 \\
25 \\
59 \\
\end{array}$ & $\begin{array}{c}28 \\
1481 \\
10 \pm 4 \\
3.0 \\
27 \\
12 \\
11 \\
\end{array}$ & \begin{tabular}{c|}
3 \\
169 \\
$1.2 \pm 0.5$ \\
1.0 \\
1.5 \\
4.2 \\
1.6 \\
\end{tabular} & $\begin{array}{l}\text { Raw promptelectrons } \\
\text { Pions in electron fiducial volume } \\
\text { Misidentified pions } \\
\text { Background from non-prompt } e^{ \pm} \\
\text {Efficiency corrected signal } \\
\text { Statistical error } \\
\text { Systematic error }\end{array}$ \\
\hline $2-3$ & $\begin{array}{c}115 \\
4104 \\
62 \pm 33 \\
16 \\
60 \\
22 \\
57 \\
\end{array}$ & $\begin{array}{c}54 \\
2633 \\
21 \pm 13 \\
6.0 \\
43 \\
14 \\
20 \\
\end{array}$ & $\begin{array}{c}21 \\
707 \\
4.9 \pm 2.1 \\
1.0 \\
25 \\
8 \\
4 \\
\end{array}$ & $\begin{array}{c}13 \\
445 \\
3.1 \pm 1.3 \\
0.5 \\
16 \\
6 \\
3 \\
\end{array}$ & $\begin{array}{l}\text { Raw prompt electrons } \\
\text { Pions in electron fiducial volume } \\
\text { Misidentified pions } \\
\text { Background from non-prompt } e^{ \pm} \\
\text {Efficiency corrected signal } \\
\text { Statistical error } \\
\text { Systematic error }\end{array}$ \\
\hline $3-4$ & $\begin{array}{c}46 \\
1911 \\
19 \pm 10 \\
7.0 \\
31 \\
13 \\
18 \\
\end{array}$ & $\begin{array}{c}31 \\
1329 \\
8 \pm 4 \\
2.0 \\
32 \\
10 \\
8 \\
\end{array}$ & $\begin{array}{c}12 \\
363 \\
1.8 \pm 0.7 \\
1.0 \\
14 \\
6 \\
2 \\
\end{array}$ & $\begin{array}{c}9 \\
269 \\
1.3 \pm 0.5 \\
0.5 \\
11 \\
5 \\
2 \\
\end{array}$ & $\begin{array}{l}\text { Raw prompt electrons } \\
\text { Pions in electron fiducial volume } \\
\text { Misidentified pions } \\
\text { Background from non-prompt } e^{ \pm} \\
\text {Efficiency corrected signal } \\
\text { Statistical error } \\
\text { Systematic error }\end{array}$ \\
\hline 45 & $\begin{array}{c}29 \\
923 \\
6.5 \pm 2.8 \\
1.0 \\
33 \\
9 \\
6 \\
\end{array}$ & $\begin{array}{c}23 \\
696 \\
3.5 \pm 2.1 \\
1.0 \\
29 \\
8 \\
4 \\
\end{array}$ & $\begin{array}{c}10 \\
231 \\
0.9 \pm 0.5 \\
0.5 \\
13 \\
5 \\
1 \\
\end{array}$ & $\begin{array}{c}6 \\
176 \\
0.7 \pm 0.4 \\
0.25 \\
8 \\
4 \\
1 \\
\end{array}$ & $\begin{array}{l}\text { Raw prompt electrons } \\
\text { Pions in electron fiducial volume } \\
\text { Misidentified pions } \\
\text { Background from non-prompt } e^{ \pm} \\
\text {Efficiency corrected signal } \\
\text { Statistical error } \\
\text { Systematic error }\end{array}$ \\
\hline $5-6$ & \begin{tabular}{|c|}
7 \\
484 \\
$3.4 \pm 1.5$ \\
0.5 \\
4.7 \\
5.0 \\
2.8 \\
\end{tabular} & $\begin{array}{c}11 \\
369 \\
1.8 \pm 1.1 \\
0.5 \\
13.2 \\
5.6 \\
1.9 \\
\end{array}$ & \begin{tabular}{|c|}
5 \\
141 \\
$0.6 \pm 0.3$ \\
0.25 \\
6.4 \\
3.7 \\
0.7 \\
\end{tabular} & $\begin{array}{c}8 \\
95 \\
0.4 \pm 0.2 \\
0.25 \\
11.2 \\
4.5 \\
0.9 \\
\end{array}$ & $\begin{array}{l}\text { Raw prompt electrons } \\
\text { Pions in electron fiducial volume } \\
\text { Misidentified pions } \\
\text { Background from non-prompt } e^{ \pm} \\
\text {Efficiency corrected signal } \\
\text { Statistical error } \\
\text { Systematic error }\end{array}$ \\
\hline$>6$ & \begin{tabular}{|c|}
9 \\
657 \\
$3.9 \pm 2.0$ \\
0.5 \\
7.0 \\
5.6 \\
3.3 \\
\end{tabular} & $\begin{array}{c}9 \\
565 \\
2.3 \pm 1.7 \\
0.5 \\
9.5 \\
5.2 \\
2.1 \\
\end{array}$ & $\begin{array}{c}4 \\
200 \\
0.6 \pm 0.4 \\
0.25 \\
4.8 \\
3.4 \\
0.7 \\
\end{array}$ & \begin{tabular}{|c|}
3 \\
228 \\
$0.7 \pm 0.5$ \\
0.25 \\
3.2 \\
3.0 \\
0.7 \\
\end{tabular} & $\begin{array}{l}\text { Raw prompt electrons } \\
\text { Pions in electron fiducial volume } \\
\text { Misidentified pions } \\
\text { Background from non-prompt } e^{ \pm} \\
\text {Efficiency corrected signal } \\
\text { Statistical error } \\
\text { Systematic error }\end{array}$ \\
\hline
\end{tabular}




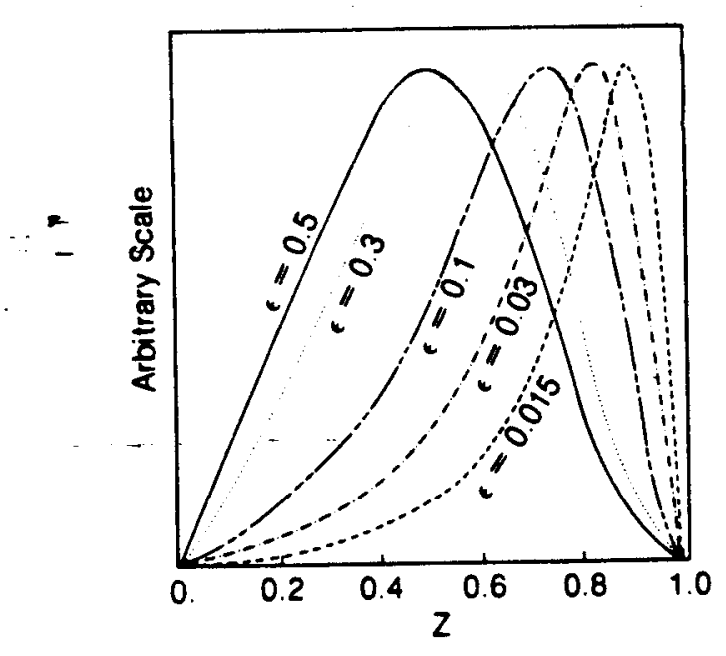

Fig. 16. Shape of $D_{Q}^{H}(z)$ for several values of $c$.

$$
\langle 2\rangle_{b}=0.75 \pm 0.05
$$

Table 11 shows the fitted populations for heavy quark production in the various $\mathrm{p}, \mathrm{PT}$ bins. The background in this table is determined directly from Table 1 . We nete that the low $p$, low $p$ T bins $\left(p<2, p_{T}<1.0\right.$ or $\mathrm{p}<3, \mathrm{p}_{\mathrm{T}}<0.5 \mathrm{GeV} / \mathrm{c}$ ) are dominated by background; they are not used in the fit. As expected, the - high $\mathrm{P}_{\mathrm{T}}$ region $\left(p_{T} \geq 1.0 \mathrm{GeV} / \mathrm{c}\right)$ is dominated by $b$ events, while lower $p_{T}$ tends to be mainly background and $c$ events. The background subtracted cross sections as a function of $\mathrm{p}$ and $\mathrm{p}_{\mathrm{T}}$ are shown in Fig. 17.

Table II. Contributions to inclusive electron production from background, charm, and bottom

\begin{tabular}{|c|c|c|c|c|c|c|c|c|}
\hline$p \backslash p_{T}$ & \multicolumn{2}{|c|}{0.0 .5} & \multicolumn{2}{|c|}{$0.5-1.0$} & \multicolumn{2}{|c|}{$1.0-1.5$} & \multicolumn{2}{|c|}{$>1.5$} \\
\hline 1.00 & & $\begin{array}{r}306.9 \\
48.7 \\
2.7 \\
\end{array}$ & 10.6 & $\begin{array}{l}68.6 \\
21.8 \\
11.7\end{array}$ & 3.1 & $\begin{array}{r}13.4 \\
4.3 \\
11.2 \\
\end{array}$ & 0.2 & $\begin{array}{l}2.2 \\
0.5 \\
0.9\end{array}$ \\
\hline 2.00 & & $\begin{array}{r}77.6 \\
24.1 \\
3.4\end{array}$ & 3.7 & $\begin{array}{l}27.1 \\
17.8 \\
11.2\end{array}$ & 1.3 & $\begin{array}{l}5.2 \\
2.0 \\
9.9 \\
\end{array}$ & 0.3 & $\begin{array}{l}3.2 \\
1.7 \\
6.1 \\
\end{array}$ \\
\hline 3.00 & 1.3 & $\begin{array}{r}26.1 \\
18.0 \\
2 .\end{array}$ & 1.5 & $\begin{array}{r}10.0 \\
13.4 \\
7.2 \\
\end{array}$ & 0.5 & $\begin{array}{l}2.8 \\
2.8 \\
6.7 \\
\end{array}$ & 0.1 & $\begin{array}{l}1.8 \\
2.3 \\
7.7 \\
\end{array}$ \\
\hline 4.00 & 0.2 & $\begin{array}{r}7.5 \\
10.2 \\
2.5 \\
\end{array}$ & 0.2 & $\begin{array}{r}4 . \\
8.0 \\
4.8 \\
\end{array}$ & 0.1 & $\begin{array}{l}1.4 \\
1.8 \\
4.3 \\
\end{array}$ & 0.0 & $\begin{array}{l}1.0 \\
1.3 \\
4.1 \\
\end{array}$ \\
\hline 5.00 & 0.1 & $\begin{array}{l}3.9 \\
5.2 \\
2.1 \\
\end{array}$ & 0.1 & $\begin{array}{l}2.3 \\
4.2 \\
3.6 \\
\end{array}$ & 0.1 & $\begin{array}{l}0.8 \\
0.7 \\
2.6 \\
\end{array}$ & 0.0 & $\begin{array}{l}0.6 \\
0.7 \\
3.2 \\
\end{array}$ \\
\hline 6.00 & 0.1 & $\begin{array}{l}4.4 \\
4.4 \\
1.7\end{array}$ & 0.1 & $\begin{array}{l}2.8 \\
4.9 \\
4.0 \\
\end{array}$ & 0.0 & $\begin{array}{l}0.8 \\
0.5 \\
4.2 \\
\end{array}$ & 0.0 & $\begin{array}{l}0.9 \\
0.0 \\
2.8\end{array}$ \\
\hline $\begin{array}{l}\text { Lege } \\
\text { are } \\
\text { charm } \\
\text { botto }\end{array}$ & $\begin{array}{l}\text { in ea } \\
\text { to } \mathrm{ev} \\
\text { om b } \\
\text { rom } \mathrm{b}\end{array}$ & $\begin{array}{l}\mathrm{p}-\mathrm{p} \text {, } \\
\text { cont } \\
\text { cay, } \\
\text { vents }\end{array}$ & $\begin{array}{l}\text { the } \\
\text { ions } \\
\text { harm }\end{array}$ & $\begin{array}{l}\text { ir valu } \\
\text { om a) } \\
\text { om cé }\end{array}$ & $\begin{array}{l}\text { n the } \\
\text { kgro } \\
\text { ents, }\end{array}$ & $\begin{array}{l}\text { yout } \\
\text {, b) } \\
\text { d d) }\end{array}$ & 0 & $\begin{array}{l}\mathrm{a} \\
\mathrm{c} \\
\mathrm{d}\end{array}$ \\
\hline
\end{tabular}



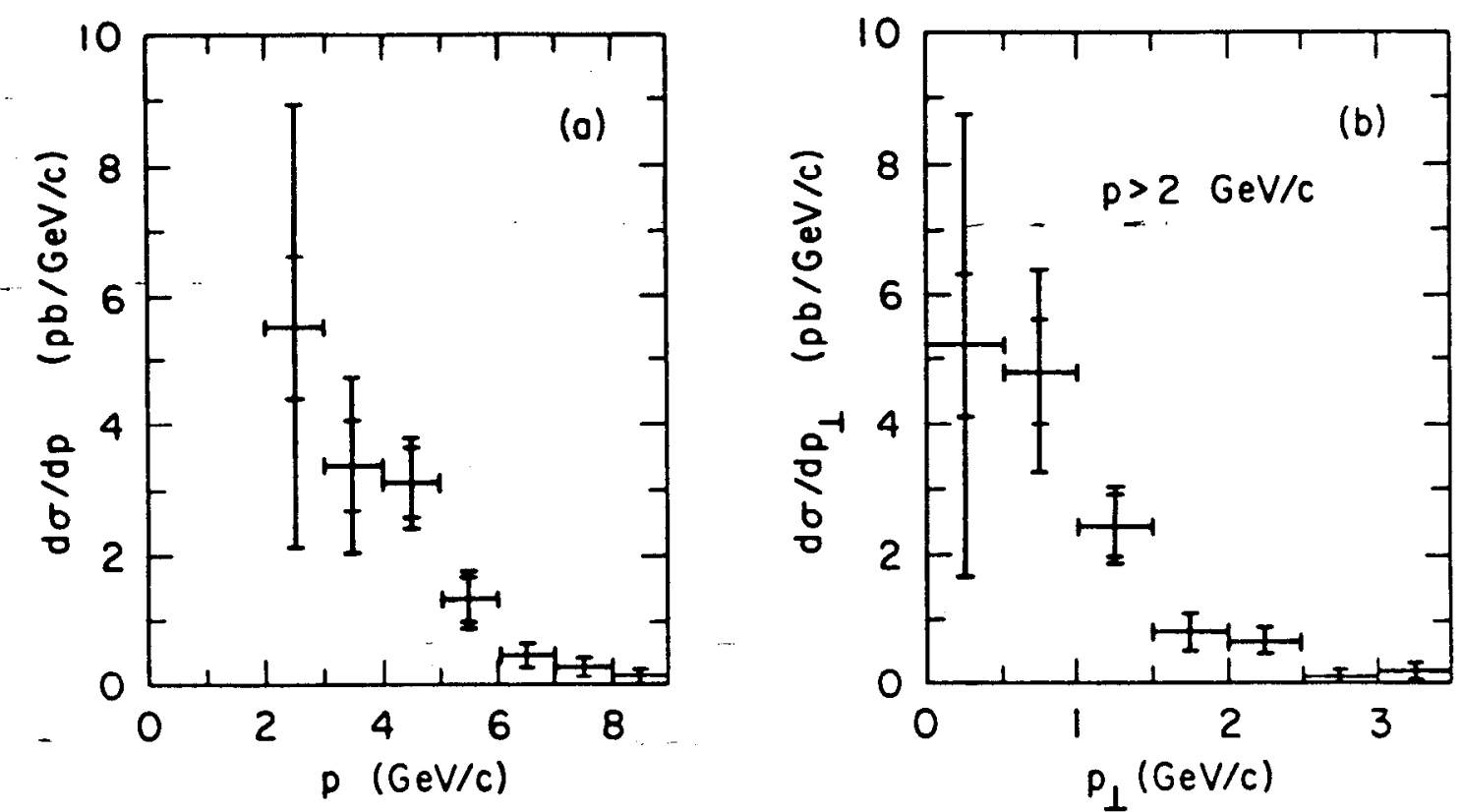

Fig. 17. Inclusive electron differential cross sections from heavy quarks $c$ and $b$ (with background subtracted).

\section{$D^{*}$ FRAGMENTATION}

The tagging of $D^{*}$ events via the decay chain

$$
\begin{aligned}
\mathrm{D}^{++} \rightarrow & \mathrm{D}^{0} \boldsymbol{\pi}^{+} \\
& \mathrm{L}^{-\mathrm{K}^{-} \pi^{+}}
\end{aligned}
$$

has been shown above to provide a clean sample of charm-associated events at SPEAR. Figure 18 shows data at PEP: the distribution in $\Delta$ is shown for two regions in 2 . A clear signal for $D^{*}$ production is seen near $145 \mathrm{MeV} / \mathrm{c}^{2}$ for $\mathrm{z}>0.4$, while for smaller $\mathrm{z}$ no signal is apparent. A Monte Carlo study of $B \rightarrow D^{\circ}+n \pi$ decays suggests that the null signal of Fig. 18(a) is consistent with a negligible contamination of $D^{\circ}$ events by the cascade from produced $b \bar{b}$ pairs.

The fragmentation function for $D^{*}$ events, selected from Fig. 18 with the cut

$$
143<\Delta<147 \mathrm{MeV} / \mathrm{c}^{2}
$$

is shown in Fig. 19. For comparison, histograms are shown for various values of $\epsilon$ : the data favor a value of $\epsilon_{\mathrm{c}} \approx 0.3$.

The inclusive lepton study of the previous section also yields information about $\epsilon_{c}$. If this parameter is allowed to vary in the overall p-pt fit, a value

$$
\epsilon_{c}=0.19+0.129
$$

is obtained. 


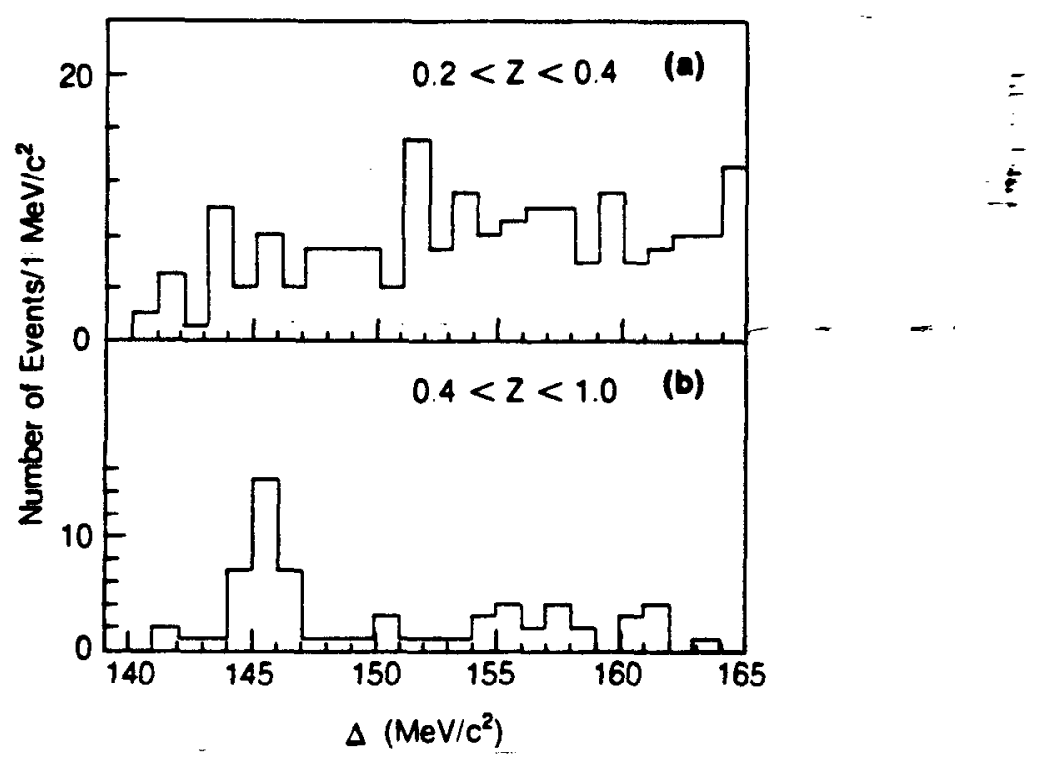

Fig. 18. Distribution of $\Delta$ for $D^{0}$ candidates at PEP.

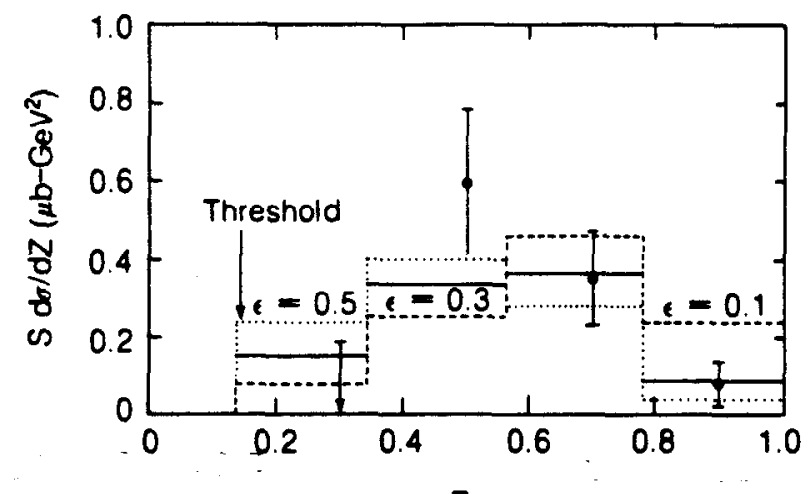

Fig. 19. Charm fragmentation function measured with $D^{*}$ events.

\section{PRODUCTION ANGULAR DISTRIBUTION FOR $b$ and $c$}

The tagged $D^{\circ}$ events with both $D^{\circ+} \rightarrow D^{0} x^{+}$and $D^{\circ+} \rightarrow S^{0} \pi^{+}$decays define a clean sample of produced cē events. These events may then be used to study the production angular distribution for $c \bar{c}$, and, in particular, to search for evidence for the axial vector coupling of the $Z^{0}$ to the $c$ quark via a forward-backward asymmetry. The angular distribution for fermionantifermion pair production may be written (far below the $Z^{\circ}$ mass)

$$
\frac{\mathrm{dn}}{\mathrm{d} \cos \theta} \sim 1+\cos ^{2} \theta+\lambda \cos \theta
$$

where is the angle between the fermion direction and the initial $e^{-}$direction. In the standard Weinberg-Salam model,

$$
\lambda_{\mathrm{f}} \approx \frac{-4 \mathrm{a}_{\mathrm{f}} \mathrm{a}_{\mathrm{f}}}{\mathrm{Q}} x
$$


where $a_{e}=2 g_{e}^{A}$ is the axial vector coupling of the electron $\left(\mathrm{a}_{\mathrm{e}}=-1\right.$ in the standard model), $a_{f}$ is the corresponding coupling of the fermion $f$ of charge $Q_{r}$, and $x$ is related to the $Z^{0}$ mass and the Fermi coupling constant $\left(x \approx-0.04\right.$ at $\mathrm{E}_{\mathrm{cm}}=29 \mathrm{GeV}$ ).

The angular distribution (preliminary data) for $D^{\circ}$ events is shown in Fig. 20. Here $\theta$ is the angle between the $D^{\circ}$ (not $\bar{D}^{\prime}$ ) and - the initial $e^{-}$direction: the charge of the $D^{\circ}$ is used in lieu of $K^{ \pm}$identification to specify the c or $\bar{c}$ content of an observed $D^{*}$. A surplus of events in the backward hemisphere is apparent: a fit to this distribution using the form (1) yields

$$
\lambda_{c}=-0.49 \pm 0.37 \text {. }
$$

From the value of $\lambda$, a value for $a_{c}$ of

$$
a_{c}=+2.0 \pm 1.5 \quad \text { (preliminary) }
$$

may be inferred, to be compared with the standard model value

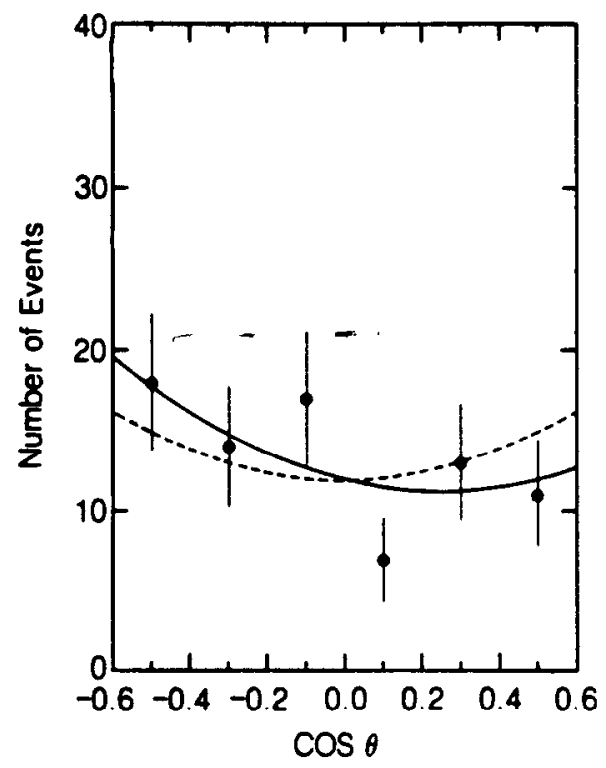

Fig. 20. Production angular distribution for $D^{*}$ events.

$$
a_{c}=+1 \quad(W-S) \text {. }
$$

A similar (and even more preliminary) analysis of the inclusive lepton data allows a measurement of the axial couplings of both the $b$ and the $c, a_{b}$ and $a_{c}$. While much refinement in the experimental method is now needed, as well as a larger statistical sample, the data are presented here as indicative of the power of the inclusive lepton tag to produce clean $b$ and $c$ events, and as illustrative of the method and of some of its problems.

The $\mathrm{p}-\mathrm{p}_{\mathrm{T}}$ plane is arbitrarily divided into two complementary regions which we label low $\mathrm{p}_{\mathrm{T}}$ and high $\mathrm{p}_{\mathrm{T}}$ in Table III. The low $\mathrm{p}_{\mathrm{T}}$ region is seen to be mainly populated by background, with $c$ dominant over $b$, while at high $\mathrm{p}_{\mathrm{T}} \mathrm{b}$ is dominant over $\mathrm{c}$ and background is smaller.

The lepton ( $e$ or $\mu$ ) charge is here used to specify the $b$ or $\bar{b}$ content of the decaying jet; the thrust axis of the event is used to specify the direction of the primeval quark. The charge signature for the $b$ is derived from the expected dominant semi-leptonic decay

$$
\mathrm{b} \rightarrow \mathrm{cl}^{-} \bar{\nu}_{l} \text {, }
$$

so that detection of an $e^{-}$or $\mu^{-}$is indicative of $b($ not $\bar{b})$ production. In contrast, the dominant $c$ decay has the opposite leptonic charge signature:

$$
c \rightarrow s l^{+} \nu_{l} \text {. }
$$

Hence in a sample of mixed $b$ and $c$ production (or of observation of $c$ quarks as a secondary product from $b$ decay), this opposite charge signature will dilute the observed asymmetry.

Table III. Separation of the inclusive lepton sample into low and high $\mathrm{PT}_{\mathrm{T}}$ classes.

\begin{tabular}{|llrrc|}
\hline Bin & Definition & b\% & $c \%$ & Background \% \\
\hline & & & & \\
low $\mathrm{pT}_{T}$ & $\mathrm{PT}_{\mathrm{T}}<1$ and $\mathrm{p}<4 \mathrm{GeV} / \mathrm{c}$ & 8 & 27 & 65 \\
high $\mathrm{pT}_{\mathrm{T}}$ & $\mathrm{PT}_{\mathrm{T}}>1$ or $\mathrm{p}>4 \mathrm{GeV} / \mathrm{c}$ & 44 & 30 & 26 \\
\hline
\end{tabular}




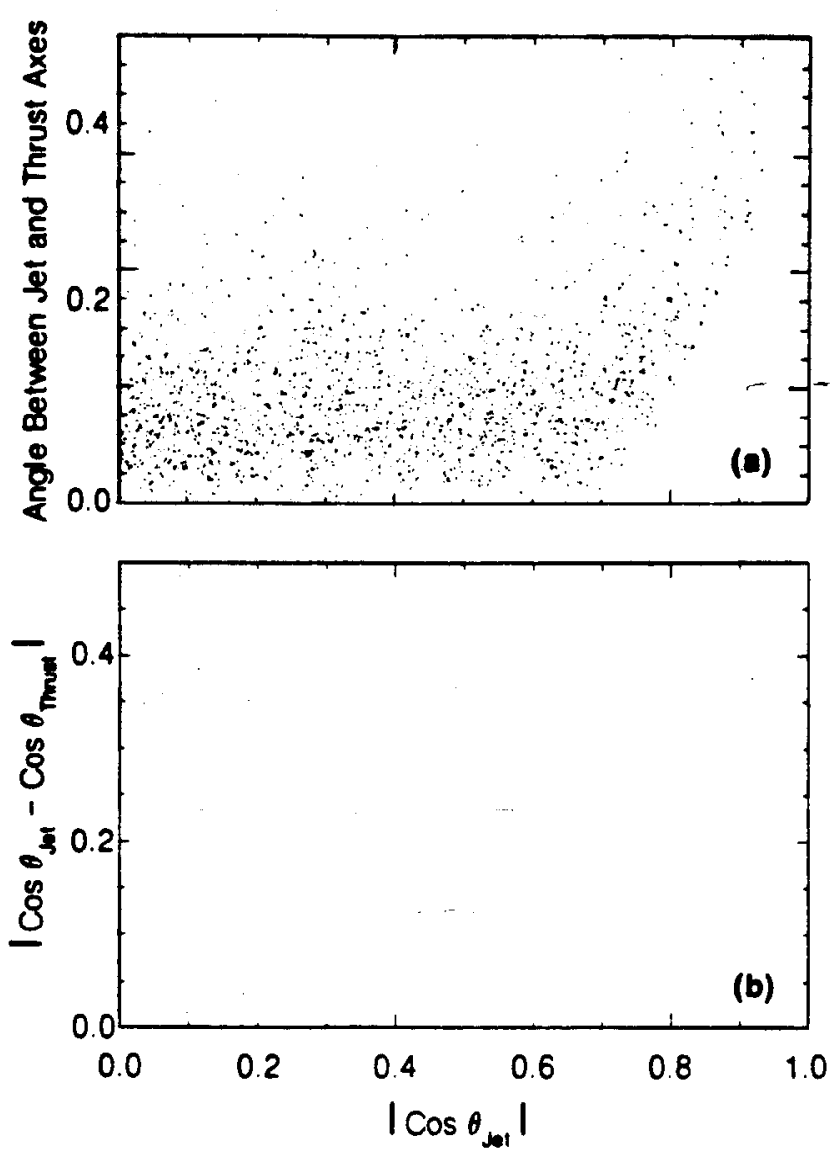

Fig. 21. Monte Carlo angular correlation studies of observed thrust and produced jet directions.

The relationship between the produced quark (jet) angular distribution and the observed thrust distribution has been studied using Monte Carlo methods. Figure 21(a) shows that for $\left|\cos \theta_{\text {Jet }}\right|<0.6, \sim 85 \%$ of the $b \bar{b}$ events have produced and measured axes that agree within $10^{\circ}$. Figure:21(b) shows that for radiative $b \bar{b}$ events, either with initial state $\gamma$ or final state gluon emission, this correlation is no longer apparent.

In Fig. 22 is shown the production angular distribution for the two pr bins of the

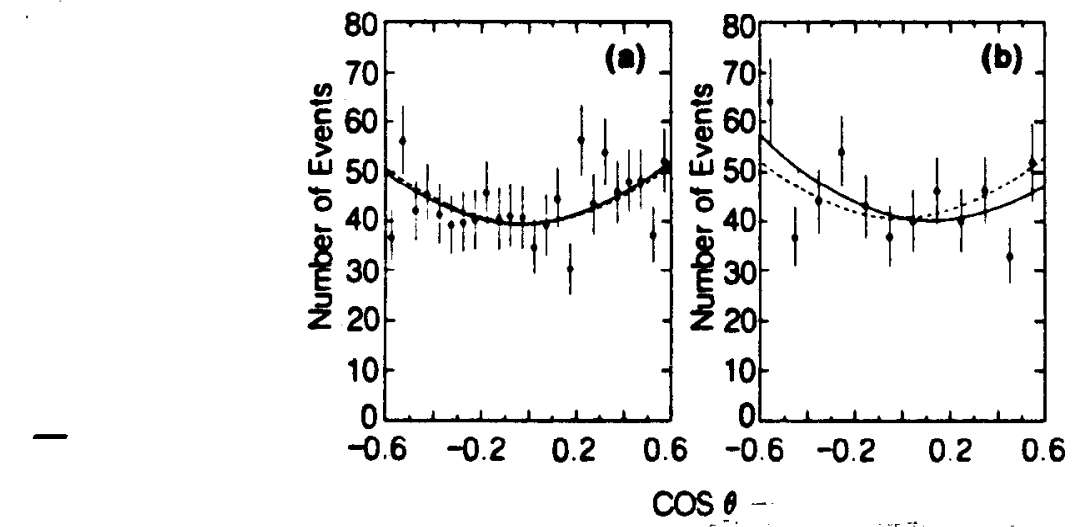

Fig. 22. Production angular distribution for inclusive lepton data: a) low $p_{T}, b$ ) high $\boldsymbol{p}_{\boldsymbol{T}}$. 
inclusive lepton sample. This distribution has been corrected for acceptance with a Monte Carlo calculation. Taking into account the relative populations of the two $\mathrm{p}_{\mathrm{T}}$ bins, we have fit the data to the form (1). The resulting fit, as well as the calculated forward-backward asymmetries, shows that the observed charge correlation is of opposite sign in Figs. 22(a) and 22(b), as would be expected for the measured $b$ and $c$ content of these two pr bins. With a Monte Carlo calculation of the dilutive effects of initial state radiation and gluon emission (estimated to be $0.7 \pm 0.2$ in the coupling $\lambda$ ), the fitted value of the $b$ axial vector coupling is (assuming $\lambda_{c}=1 / 2 \lambda_{b}$; the factor of $1 / 2$ is due to the larger charge, $2 / 3$, of the $c$ quark than the $Q=1 / 3 b$ charge)

to be compared with

$$
a_{b}=-3.0 \pm 2.1 \quad \text { (preliminary) }
$$

$$
a_{b}=-1 \quad(W-S)
$$

\section{ACKNOWLEDGMENT}

This work was supported in part by the Director, Office of Energy Research, Division of High Energy and Nuclear Physics of the of the U.S. Department of Energy under contracts DEAC03-76SF00098 and DE-AC03-76SF00515.

\section{REFERENCES}

1. W. Davies-White et al., Nuclear Instr. and Methods 160, 227 (1979).

2. G.S. Abrams, IEEE Trans. Nucl. Sci. NS27, 59 (1980).

3. G. Feldman Phys. Rev. Letters 38, 1313 (1977).

4. This work is mainly that of Gerson Goldhaber (see TG-350, March 19, 1982).

5. - C. Peterson, D. Schlatter, I. Schmitt, and P.M. Zorwar, SLAC-PUB-2912.

6. K. Chadwick et al., CLNS-82-546.

7. W. Bacino et al., Phys. Rev. Lett. 43, 1073 (1979).

\section{DISCUSSION}

Robert I. Hulsizer, MIT:

Was the quantity $\Delta=M\left(D^{*}\right)-M(D)$ calculated on an event-by-event basis?

Abrams: Yes, and that is the crucial point in this analysis. The mass errors in the $D$ and $D^{*}$ are strongly correlated. Taking the difference of the two measured masses eliminates much of the effect of momentum measurement error, and produces the narrower signal in $\Delta$. 